\title{
Kurzbeitrag
}

\section{Zusammenarbeit von Arbeitsämter und Kommunen ausbauen}

90 Prozent aller Arbeits- und Sozialämter halten eine intensivere Kooperation für notwendig (Bertelsmann Stiftung u.a. 2001). Denn viele Integrationsprobleme lassen sich durch eine bessere $\mathrm{Zu}$ sammenarbeit leichter lösen (Bertelsmann Stiftung u.a. 2000). Dabei ist es zweitrangig, von welchem Amt der Hilfeempfänger Leistungen erhält - wichtig ist, das schnell die richtige Hilfe angeboten wird. Viele Arbeits- und Sozialämter arbeiten heute schon gut zusammen. Seit 1. Dezember 2000 ist diese Zusammenarbeit nun auch gesetzlich vorgeschrieben. Doch wie soll die $\mathrm{Zu}-$ sammenarbeit geplant und umgesetzt werden?

\section{Kooperation rechnet sich}

Es gibt fünf gute Gründe für die Zusammenarbeit von Arbeitsämtern und Kommunen:

- Erhöhung der Integrationschancen durch Bündelung von Kompetenzen

Die Integration der Hilfeempfänger ist eine anspruchsvolle Aufgabe. Die Vermittlungshemmnisse, die durch langjährige Arbeitslosigkeit entstanden sind, können nur durch maßgeschneiderte Hilfen beseitigt werden. Dabei müssen berufliche und soziale Voraussetzungen berücksichtigt werden. Durch eine Bündelung der unterschiedlichen Kompetenzen beider Ämter wird dies gewährleistet - die Integrationschancen erhöhen sich so deutlich.

- Die Reduzierung von Doppelarbeit durch effiziente Arbeitsteilung

Wenn Personen von beiden Ämtern Leistungen beziehen, müssen für ein und denselben Hilfeempfänger viele Arbeiten zweimal erledigt werden - die Aufnahme der Hilfeempfängerdaten, die Berechnung der Unterstützungszahlungen, Beratungsgespräche usw. Eine Kooperation bietet hier viele Möglichkeiten, mittelfristig die Doppelarbeiten signifikant zu reduzieren.
- Wachsende Mitarbeiterzufriedenheit durch Know-How-Transfer

Mehr Erfolg bedeutet mehr Zufriedenheit. Dafür können die höheren Integrationsquoten sorgen, die für das Image beider Institutionen gut sind. Der Know-how-Transfer zwischen den Mitarbeitern der Ämter wird zudem das Spektrum an Handlungsmöglichkeiten erweitern. Einen weiteren Beitrag zur wachsenden Mitarbeiterzufriedenheit leistet die Reduzierung der Doppelarbeiten.

- Mehr Bürgernähe durch Konzentration auf Integrationshilfen

Was für die Ämter zweifache Arbeit bedeutet, ist aus der Sicht des Hilfeempfängers ein „Wildwuchs von Bürokratie“: zwei Stellen, zwei Anträge zweifache Schilderung der sozialen Situation. Die Zusammenarbeit kann dies ändern. Der Bürger wird sich dadurch in seiner Notlage ernst genommen fühlen und eher bereit sein, seinen Anteil an der Integrationsarbeit zu leisten.

- Kostenersparnis durch Synergieeffekte Durch Synergieeffekte sowie deutlich verbesserte Integrationsquoten werden beide Ämter mittelfristig und nachhaltig eine Menge Geld einsparen. Doch zuerst muss man investieren - und mit mehr Arbeit und höheren Ausgaben rechnen.

\section{Informationsaustausch - Kooperation braucht Vertrauen}

Der gute Informationsaustausch zwischen den Ämtern erfüllt vor allem die Funktion, die Transparenz über Ziele, Ressourcen und Personen des jeweiligen Amtes herzustellen. Wenn dies regelmäßig, zeitnah und vollständig geschieht - auf Führungs- und Sachbearbeiterebene und selbstverständlich gegenüber der kommunalen Spitze und der Selbstverwaltung des Arbeitsamtes - ist der Informationsaustausch einer der wichtigsten Bausteine für die vertrauensvolle Kooperation. (Marburg, Heilbronn: Die Ortsnamen verweisen 
auf Umsetzungsbeispiele, die im Handbuch dokumentiert sind.)

\section{Finanzierung - unterschiedliche Quellen, gemeinsame Ziele}

Die Möglichkeiten des Finanzierungsmixes für Doppelbezieher werden inzwischen von vielen Ämtern genutzt. In Osnabrück werden die unterschiedlichen Maßnahmemöglichkeiten des Arbeitsamtes benannt und - das ist entscheidend - die Verfahren aufgezeigt, wie Sozialhilfeempfängern (mit und ohne Leistungsbezug durch das Arbeitsamt) in diese integriert werden können. Auch die Zuständigkeiten werden definiert. Mit dem modular aufgebauten Hilfsangebot der MAVA in Mannheim können Maßnahmen der beiden Ämter passgenau für den jeweiligen Hilfeempfänger aufeinander abgestimmt werden. So kann der Förderbaustein, der das Selbsthilfepotential des Hilfeempfängers am geschicktesten ausnutzt, auch zum richtigen Zeitpunkt zum Einsatz kommen.

Wichtig ist, dass die gemeinsame Finanzierung vertraglich abgesichert ist. Es sollte festgelegt sein, wessen Geld wann und wie eingesetzt wird. Die Finanzierungsregeln werden durch klare Protokollierungen ,prüfungsfest“.

\section{$4 \quad$ Austausch der \\ Hilfeempfängerdaten - Reduzierung der Doppelarbeit}

Aus der Sicht beider Ämter ist der beschwerdefreie Austausch derHilfeempfängerdaten die wichtigste Voraussetzung einer Erfolg versprechenden Kooperation. Ziel sollte es sein, dass nicht die Menschen, sondern die Daten zwischen den Ämtern laufen. Der uneingeschränkte Datenaustausch ist möglich - wenn der Hilfeempfänger dazu seine schriftliche Einverständniserklärung gibt. In der gemeinsamen Stelle in Pirmasens, in der Mitarbeiter beider Ämter abgeordnet sind, kann es daher einen gemeinsamen Erfassungsbogen und eine Datenbank geben.

Auch empfehlenswert ist das Verfahren der Jobbörsen in Köln. Dort halten die Mitarbeiter regelmäßig eine gemeinsame, im Einzelfall vereinbarte Fallkonferenz ab.

Für Erfolg versprechende Integrationsmaßnahmen können 7 Qualitätskriterien aufgestellt werden, die sich bei vielen Beispielen bewährt haben.
- Fall-Management: Der Hilfeempfänger hat einen Ansprechpartner, der alle Maßnahmen koordiniert $(K o ̈ l n)$

- Der Hilfeplan (auch Eingliederungs- oder Entwicklungsplan) dokumentiert verbindlich die jeweiligen Aufgaben. Erfolge und Misserfolge können so überprüft werden. (Krefeld)

- Arbeitsmarktnähe: In Gesprächen zwischen Arbeitgebern, Verbänden und Kammern können Bedarfe identifiziert und Wege erkundet werden, wie Arbeitslose Jobs bekommen können.

- Sofortige Integration in den Arbeitsmarkt ist dem Hilfebezug vorzuziehen. Pirmasens versucht, jeden Antragsteller umgehend zu vermitteln.

- Ressourcen- statt Maßnahmenorientierung: die Maßnahmen müssen auf die Stärken/ Schwächen der Hilfeempfänger zugeschnitten werden.

- Fördern und Fordern, die Balance zwischen staatlichen Hilfen und der Eigeninitiative und Mitwirkung des Hilfeempfängers. (Münster)

- Evaluation: Eine regelmäßige und fundierte Evaluation von Maßnahmen ist unabdingbare Voraussetzung für eine erfolgreiche Steuerung.

\section{Gemeinsame Anlauf- und Beratungsstellen}

Eine gemeinsame Anlauf- und Beratungsstelle beider Ämter einzurichten ist sicherlich die anspruchsvollste Form der Kooperation. Aber diese Mühe lohnt sich, denn die Erfahrungen zeigen, dass durch eine gemeinsame Stelle gleich mehrere Probleme behoben werden können: Für die Klienten entfallen die doppelten Wege, und auch die Mitarbeiter werden von doppelten Arbeiten entlastet. Die Hilfeinstrumente beider Ämter werden von einer Stelle ausgehend organisiert, so dass passgenaue und marktgerechte Hilfemaßnahmen angeboten und finanziert werden können. Für die Arbeitsämter ist es von großer Bedeutung, dass die gemeinsamen Anlaufstellen mit der Philosophie des neuen Organisationsmodells „Arbeitsamt 2000“kompatibel sind. (Magdeburg, Marburg, Göttingen) 


\section{Zusammenarbeit mit Trägern und beauftragten Dritten}

Beide Ämter arbeiten bereits in vielfältiger Weise mit Trägern zusammen, deren Leistungen sie einkaufen. Die Steuerung und Kontrolle der Maßnahmenträger ist eine schwierige Aufgabe. Zuerst sollten die Träger über transparente Qualitätskriterien ausgewählt werden. Ein präzises Anforderungsprofil sollte in die Ausschreibung eingehen mit dem Ziel, Aufgaben von vornherein transparent und verbindlich zu gestalten. Um die Ergebnisorientierung der Träger zu verbessern, sollten diese mittelfristig nicht mehr nur durch Festbetragsfinanzierung, sondern auch über leistungsorientierte Prämien honoriert werden (Berlin). Um die Kontroll- und Steuerungsmöglichkeiten gegenüber dem Träger weiter zu verbessern, sollten im Rahmen eines DV-gestützten ManagementInformations-Systems (MIS) Kennzahlen Transparenz über den Grad der Zielerreichung liefern. In Krefeld werden Dritte mit der Begleitung des Reintegrationsprozesses durch Fall-Manager beauftragt. Seit 1998 können die Arbeitsämter Vermittlungsaufgaben delegieren. Der Einkauf von Dienstleistungen sollte als Entlastung der Mitarbeiter und Ergänzung der Hilfeangebote gedacht und kommuniziert werden.

\section{$7 \quad$ Vertraglich geregelte Zusammenarbeit}

Ein Kooperationsvertrag macht die Ziele der Kooperation für alle Beteiligten transparent. Transparenz ist Voraussetzung für Vertrauen. Insofern ist der Vertrag ein Baustein des Vertrauens zwischen den Ämtern. Des Weiteren wird durch den Vertrag die Kooperation verbindlich. Es entsteht Sicherheit für die Partner. So sollte ein Rahmenvertrag die wichtigsten Leitlinien für die Kooperation festlegen. Für Präzisierungen und Ergänzungen dienen dann eine gemeinsame Jahresplanung und/oder Umsetzungsvereinbarungen (Bertelsmann Stiftung u.a. 2001). Es empfiehlt sich, den Verträgen im vornherein eine begrenzte Dauer zu verleihen. Ein fortzuschreibender Vertrag kann flexibel auf veränderte Rahmenbedingungen reagieren.

Die Partner sollten regelmäßig die Öffentlichkeit über Ihre Planungen und Ergebnisse informieren. Eine gemeinsame PR lässt bei niemanden den Eindruck aufkommen, einer der beiden
Partner hätte mehr mit dem Erfolg zu tun als der andere. Der Erfolg gehört beiden Partnern - zur Freude der „Kunden“ - Erwerbslose und Arbeitgeber.

\section{Literatur}

Bertelsmann Stiftung, Bundesanstalt für Arbeit, Deutscher Landkreistag, Deutscher Städtetag, Deutscher Städte- und Gemeindebund (Hg.) (2001): Handbuch zur Kooperation von Arbeitsämtern und Kommunen. Gemeinsam für die Integration in den Arbeitsmarkt. Gütersloh (im internet: www.bikonline.de)

Bertelsmann Stiftung (Hg.) (2000): ,Kooperation statt Konkurrenz“, Gütersloh 2000 (download unter: www.stiftung.bertelsmann.de/Publikationen/ Downloads/Wirtschaft)

\section{Frank Frick}

Leiter Abteilung Arbeitsmarkt und Beschäftigung Bertelsmann Stiftung

Bereich Wirtschaft

Carl-Bertelsmann-Str. 256

D-33311 Gütersloh 